\title{
Identification of Two Novel DNAAF2 Variants in Two Consanguineous Families with Primary Ciliary Dyskinesia
}

\author{
Chenyang Lu (iD ${ }^{1-3, *}$ \\ Danhui Yang ${ }^{1-3, *}$ \\ Cheng Lei ${ }^{1-3}$ \\ Rongchun Wang ${ }^{1-3}$ \\ Ting Guo ${ }^{1-3}$ \\ Hong Luo (D) ${ }^{1-3}$
}

'Department of Pulmonary and Critical Care Medicine, The Second Xiangya Hospital, Central South University, Changsha, People's Republic of China;

${ }^{2}$ Research Unit of Respiratory Disease, Central South University, Changsha, People's Republic of China; ${ }^{3}$ Hunan Diagnosis and Treatment Center of Respiratory Disease, Changsha, People's Republic of China

*These authors contributed equally to this work
Correspondence: Hong Luo; Ting Guo Department of Pulmonary and Critical Care Medicine, The Second Xiangya Hospital, Central South University, 139 Renmin Middle Road, Furong District, Changsha, Hunan, 4I00II, People's

Republic of China

Email luohonghuxi@csu.edu.cn;

guotingxy@csu.edu.cn
Background: Dynein axonemal assembly factor 2 (DNAAF2) is involved in the early preassembly of dynein in the cytoplasm, which is essential for motile cilia function. Primary ciliary dyskinesia (PCD) associated with $D N A A F 2$ variants has rarely been reported in females with infertility. Moreover, there is no report linking DNAAF2 to scoliosis in human.

Materials and Methods: We recruited patients from two consanguineous families with a clinical diagnosis of PCD and collected their clinical history, laboratory tests, and radiographic data. Sequencing and bioinformatics analysis were then performed. Immunofluorescence and high-speed microscope analysis were used to support the pathogenicity of the variant.

Results: Proband 1, a 26-year-old female from family I, exhibited scoliosis, bronchiectasis, sinusitis, situs inversus, and infertility. We found a novel homozygous missense variant in $D N A A F 2$, c.491T>C, p.(Leu164Pro) in this patient. Subsequent immunofluorescence indicated the absence of outer dynein arm and inner dynein arm of cilia, and high-speed microscopy analysis showed that the most of the cilia are static, which support the pathogenicity of this variant. Proband 2, a 53-year-old female, presented with bronchiectasis, sinusitis, and infertility. In this patient, a new homozygous frameshift variant $D N A A F 2$, c.822del, p.(Ala275Profs*10) was identified. The disease-causing variants mentioned above are not included in the current authorized genetic databases.

Conclusion: Our findings expand the spectrum of $D N A A F 2$ variants and link $D N A A F 2$ to female infertility and likely scoliosis in patients with PCD.

Keywords: primary ciliary dyskinesia, scoliosis, $D N A A F 2$, bronchiectasis, situs inversus, female infertility

\section{Introduction}

Primary ciliary dyskinesia (PCD, MIM:244400) is a rare genetically heterogeneous disorder. ${ }^{1}$ It has a variety of clinical manifestations, such as chronic otorhinolaryngological diseases, male and/or female infertility and laterality defects, etc. ${ }^{2,3}$ More than 50 genes have reported to cause $\mathrm{PCD},{ }^{4}$ but detailed clinical evaluation has rarely conducted in adult patients with PCD, especially the phenotype associated with infertility. ${ }^{5}$

Changes in motile cilia are closely associated with PCD, and recent studies have shown that dysfunction of ependymal motile cilia can influence the flow of cerebrospinal fluid and possibly result in spinal dysplasia, eventually leading to 
scoliosis in zebrafish. ${ }^{6}$ Scoliosis is defined as a spinal deformity in a skeletally mature patient with a Cobb angle of more than 10 degrees in the coronal plane. ${ }^{7}$ Dynein axonemal assembly factor 2 (DNAAF2), involved in the early preassembly of dynein in the cytoplasm, is essential for motile cilia function ${ }^{8,9}$ and may be related to adult scoliosis.

Here, we analyzed the exome and clinical data of two individuals who were diagnosed with PCD. In our study, we validate the pathogenicity of a missense variant through immunofluorescence and high-speed microscope analysis, and link $D N A A F 2$ variants to female infertility and likely scoliosis.

\section{Materials and Methods}

\section{Ethical Compliance}

The Review Board of the Second Xiangya Hospital of Central South University in China approved the study protocol. This study was conducted in accordance with

A

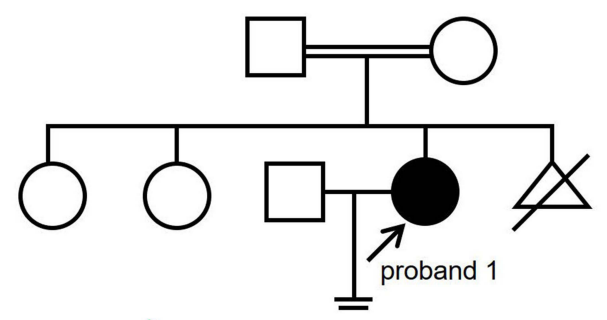

Father $\mathrm{A} \cap \mathrm{AnVM}$

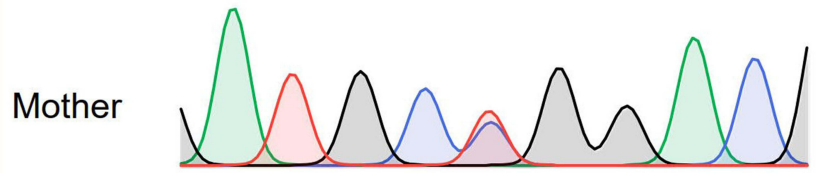

proband1 $\mathrm{A} \cap \mathrm{A} \cap \mathrm{V}$

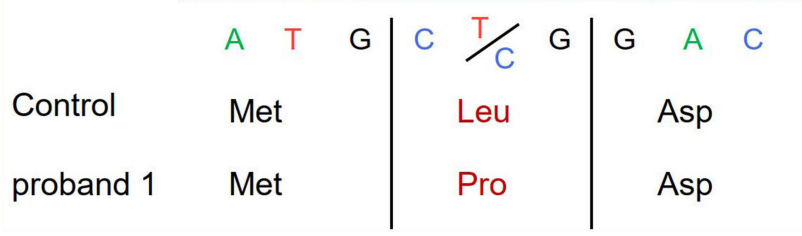

the Declaration of Helsinki. The patients and their parents were given informed consent. All experiments were strictly following the relevant guidelines and provisions. We recruited patients coming from two consanguineous families. The clinical data of the subjects, including nasal nitric oxide (nNO) level, computed tomography (CT) and chest radiographs were collected for reviewing.

\section{Measurement of $\mathrm{nNO}$}

Nasal NO was measured using an electrochemical NO analyzer, Sunvou-CA2122 nitric oxide analyzer (Sunvou, China). Patients maintain positive oral pressure by blowing into a mouth resistor as described. ${ }^{10}$ The sensor converted the NO content of the nasal sampling into current and then the concentration of NO was calculated. We converted NO concentration into NO production rate by following equation: $\mathrm{nNO}(\mathrm{nL} / \mathrm{min})=\mathrm{NO}(\mathrm{ppb}) \times$ samplingrate $(\mathrm{mL} / \mathrm{min})$.

B

Family 2
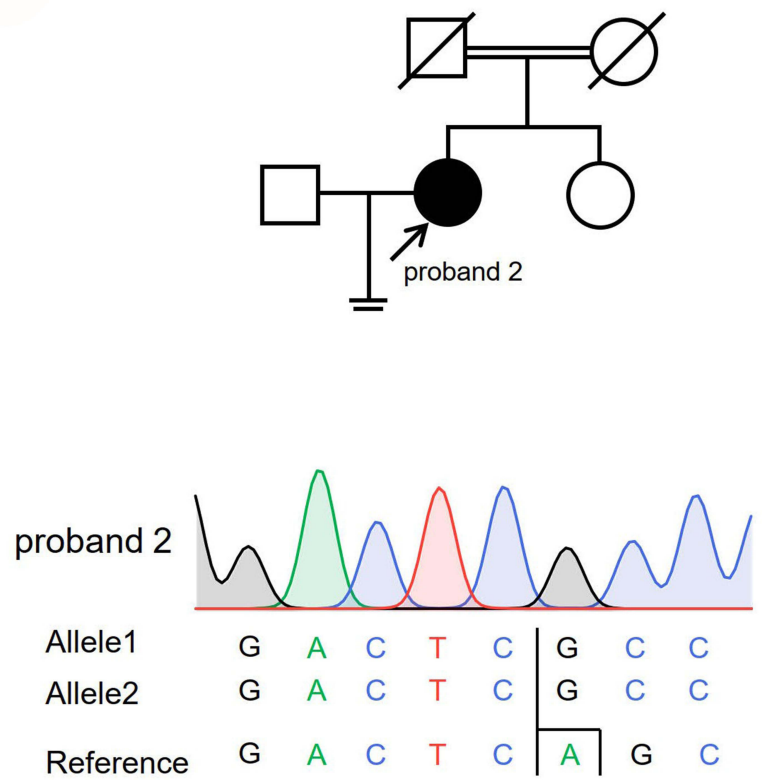

Figure I (A) Pedigree of the family I and Sanger sequencing chromatogram of proband I and her parents. A novel variant DNAAF2 c.4I9T>C, p.(Leu I64Pro) was identified in the proband. Her parents were heterozygous at the same position. (B) Pedigree of the family 2 and Sanger DNA sequencing chromatogram of proband 2 . A novel variant DNAAF2 c.822delA, p.(Ala275Profs*10) was identified in the proband. Circles indicate to females. Squares indicate males. Solid symbols indicate patients. Crossed-out symbols mean that subjects had passed away. The arrows indicate the probands. The vertical line with 2 hash marks at the end indicates infertility. 


\section{Variant Analysis}

Blood samples $(3-5 \mathrm{~mL})$ were collected from the probands and their relatives. We used the DNeasy Blood \& Tissue Kit (Qiagen, Valencia, CA) to extract genomic DNA. The Novogene Bioinformatics Institute (Beijing, China) carried out the Whole-exome capture and high-throughput sequencing. The Agilent SureSelect Human All Exon V6 kit (Agilent, California, USA) was used to set up the library and capture the whole exomes. High-throughput and high-depth sequencing was performed on Illumina platform. Sequencing reads were mapped to the human reference genome (GRCh37/ hg19). ANNOVAR software ${ }^{11}$ was used to annotate the data. Then, we classified the variants referring to the standards and guidelines for the interpretation of sequence variations from The American College of Medical Genomics (ACMG). ${ }^{12}$ Pathogenicity screening was performed on the singlenucleotide variants (SNVs)/insertion and deletions (InDels) information detected by basic analysis: 1) High-frequency variants (minor allele frequency $>0.01$ ) in 1000 genome data (1000G_ALL), ESP6500 database (ESP6500si_all), gnomAD data (gnomAD_ALL and gnomAD_EAS) were removed to obtain rare variants. 2) Preserve variants in coding regions (exonic) or splicing site regions (splicing, up and down 10bp). 3) SIFT, Polyphen-2, MutationTaster and CADD were used to screen non-synonymous SNVs. Sanger sequencing was used to validate the candidate variants. The primer sequences for Sanger sequencing were as follows: proband 1 , forward 5'-GTGGAAGTGCGGTTCGT-3' revers 5'TATACTTGGCCTTCAGGGTCT-3'; proband 2, forward 5'GCCGTCGAGAAGCAGTT-3' reverse 5'-GTAGTCAGG TTTCCTCGAGTC-3'.

\section{Immunofluorescence and High-Speed Microscopy Analysis}

Nasal brushing sample was suspended in Gibco Medium 199 (12350039 Gibco). We used anti-acetylated tubulin monoclonal antibody (T7451, 1:500, Sigma-Aldrich, Missouri, USA) to mark the ciliary axoneme, DNAH5 (HPA037470, 1:100, Sigma-Aldrich, Missouri, USA) to label the outer dynein arm (ODA), DNALI1 (HPA028305, 1:10, SigmaAldrich, Missouri, USA) to label the inner dynein arm (IDA) and DAPI to label the nuclei. Immunofluorescence was used to profile the ciliary structure of patients with $D N A A F 2$ variants. Cilia beating video was recorded at 500 frames/second at room temperature by scientific complementary metal oxide semiconductor (sCMOS) camera (Prime BSI, Teledyne Photometrics Inc, USA) attached to an upright Olympus BX53 microscope (Olympus, Tokyo, Japan) with a $40 \times$ objective.

\section{Homozygosity Mapping}

We use a high-performance homozygosity mapping tool ${ }^{13}$ and next-generation sequencing data of proband 1 and proband 2 to map the homozygous gene distribution.
A

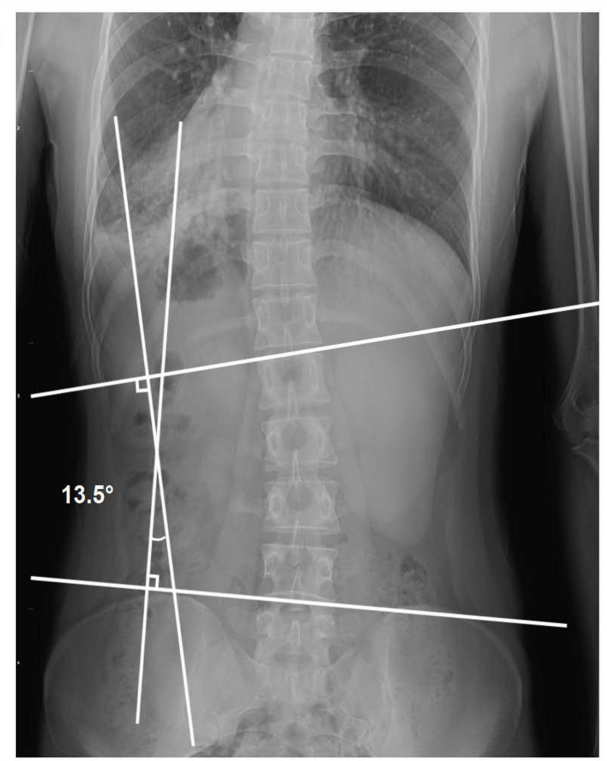

B

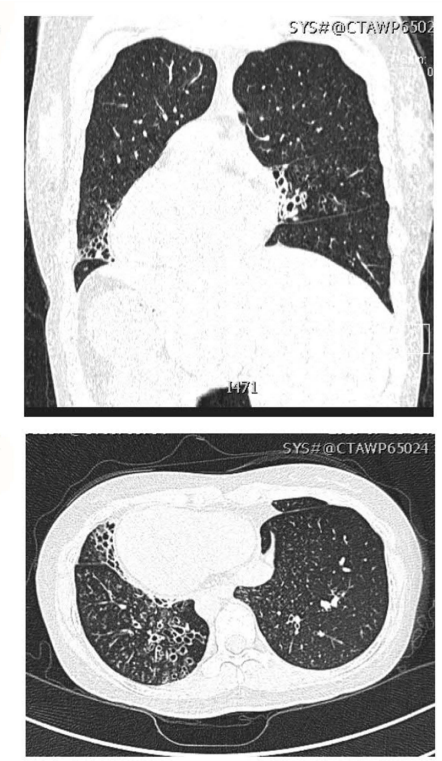

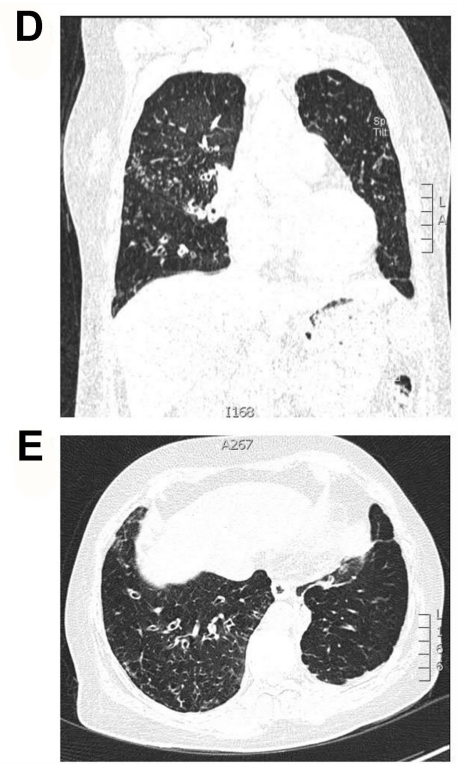

Figure 2 Clinical features of two patients in two consanguineous families. (A) Radiographic image of proband I with bronchiectasis showed right left-sided lumbar scoliosis with a Cobb's angle of $13.5^{\circ}$. (B and $\mathbf{C}$ ) The chest high-resolution computed tomography (HRCT) scan of proband I showed bronchiectasis and the situs inversus. (D and $\mathbf{E}$ ) The chest HRCT scan of proband 2 showed bronchiectasis. 
Table I Summary of the Clinical Features of Two Patients with PCD

\begin{tabular}{|c|c|c|}
\hline Characteristics & Proband I & Proband 2 \\
\hline Sex & Female & Female \\
\hline Age (years) & 26 & 53 \\
\hline Onset & Childhood & Unknown \\
\hline Consanguinity & Yes & Yes \\
\hline Inbreeding coefficient & $1 / 16$ & $1 / 16$ \\
\hline CT & $\begin{array}{l}\text { Bronchiectasis } \\
\text { Rhinosinusitis } \\
\text { Situs inversus }\end{array}$ & $\begin{array}{l}\text { Bronchiectasis } \\
\text { Rhinosinusitis }\end{array}$ \\
\hline Fertility problems & Yes & Yes \\
\hline Smell problem & Yes & Unknown \\
\hline Hearing problems & No & Unknown \\
\hline Comorbidities & Scoliosis & No \\
\hline FEVI\%prediction & $76 \%$ & NA \\
\hline $\mathrm{FEVI} / \mathrm{FVC}$ & $72 \%$ & NA \\
\hline Cobb's angle & $13.5^{\circ}$ & NA \\
\hline Nasal NO (nL/min) & 25.2 & 2.4 \\
\hline
\end{tabular}

Abbreviations: FEVI, forced expiratory volume in I second; FVC, forced vital capacity; Nasal NO, nasal nitric oxide measurement; NA, not available.

\section{Results}

\section{Clinical Summary}

Proband 1, a 26-year-old female from consanguineous family-I (Figure 1A, Supplementary Figure 1), had scoliosis, bronchiectasis, sinusitis, situs inversus and infertility. She denied any history of asthma, immunodeficiency, measles, tuberculosis, etc. Her family members had no similar symptoms or history of respiratory disease. Highresolution CT (HRCT) revealed diffuse bronchiectasis and situs inversus and upright radiograph indicated a Cobb's angle of $13.5^{\circ}$ (Figure 2A-C). Her nasal NO output level was $25.2 \mathrm{~nL} / \mathrm{min}$ (Table 1). Proband 2, a 53-year-old female from consanguineous family-II (Figure 1B), had bronchiectasis (Figure 2D and E), sinusitis and infertility. HRCT revealed diffuse bronchiectasis. The nasal NO output level was $2.4 \mathrm{~nL} / \mathrm{min}$ (Table 1).

\section{Whole-Exome Sequencing and Functional Study for the DNAAF2 Variants}

More than $10 \mathrm{~Gb}$ data were generated for each sample after whole-exome sequencing (WES). Appropriately $99 \%$ of the gene with a depth of more than $10 \times$ were mapped to the reference genes. Through alignment and frequency filtering, we further analyzed the non-synonymous variants (SNVs and InDels) and variants in exons or in canonical splice sites (splicing junction $10 \mathrm{bp}$ ). Then, we filtered the homozygous SNVs and InDels. Ultimately, we screened two different homozygous DNAAF2 variants, NC_000014.8:g.50101377A>G, NM_018139.3:c.491T >C, NP_001077377.1:p.(Leu164Pro) and NC_000014.8: g.50101046del, NM_018139.3: c.822del, NP_001077377.1: p.(Ala275Profs*10) from two consanguineous families. The missense variant was predicted to be disease-causing by SIFT, Polyphen-2, MutationTaster and CADD. The two variants were classified as likely pathogenic according to ACMG guideline (Table 2). Both variants were consistent with the family inheritance pattern, and verified by Sanger sequencing (Figure 1). Segregation of the variant $D N A A F 2$, c.491T $>$ C, p.(Leu164Pro) in two healthy sisters of proband 1 was also validated by Sanger sequencing (Supplementary Figure 1). Homozygosity mapping confirmed the homozygous region in chromosome 14 contains the variants of $D N A A F 2$ in proband 1 and proband 2 (Figure 3). Immunofluorescence of nasal ciliated cells of proband 1 revealed the absence of ODA (Figure 4). Highspeed microscopy showed that the most of the nasal cilia are static in Proband1 compared with the normal control (Supplementary Video 1 and Supplementary Video 2).

Table 2 DNAAF2 Variants Identified by Exome Sequencing for the Two Families

\begin{tabular}{|l|l|l|l|l|l|l|l|l|l|}
\hline Family & Gene & NM & $\begin{array}{l}\text { Base } \\
\text { Change }\end{array}$ & $\begin{array}{l}\text { Amino Acids } \\
\text { Change }\end{array}$ & MutationTaster & SIFT & $\begin{array}{l}\text { Polyphen- } \\
\mathbf{2}\end{array}$ & CADD & ACMG \\
\hline I & DNAAF2 & NM_018I39.3 & c.49IT>C & P.(Leul64Pro) & Disease-causing & Damaging & $\begin{array}{l}\text { Disease- } \\
\text { causing }\end{array}$ & $\begin{array}{l}25.6 \\
\text { PS3+PM2 } \\
+P P I+P P 3\end{array}$ \\
\hline 2 & DNAAF2 & NM_018I39.3 & c.822del & $\begin{array}{l}\text { P. } \\
\text { (Ala275Proff*10) }\end{array}$ & NA & NA & NA & NA & PVSI+PM2 \\
\hline
\end{tabular}

Abbreviation: NA, not available. 


\section{Discussion}

In this study, whole-exome sequencing combined with homozygosity mapping was used to identify homozygous variants in $D N A A F$ in two consanguineous families. The two novel variants were validated to be disease causing by using immunofluorescence and high-speed microscope analysis. These two female patients presented with infertility; moreover, upright radiograph in one patient revealed scoliosis.

PCD is a genetic ciliopathy caused by dysfunction of respiratory motile cilia and failure of mucociliary clearance. ${ }^{14}$ The respiratory manifestations include neonatal respiratory distress, chronic rhinosinusitis, otitis media, and bronchiectasis. Cilia in other organs can be involved, such as embryonic node, female fallopian tubes, sperm, and ependymal cells.

$D N A A F 2$ is involved in the early preassembly of dynein in the cytoplasm, which is essential for motile cilia function. ${ }^{2,8,9,15}$ Deficiency of the protein can lead to loss of inner and outer dynein in cilia axoneme, and cilia beating defect. Multiple $D N A A F 2$ variants: c. $23 \mathrm{C}>\mathrm{A}$, c. $26 \mathrm{C}>\mathrm{A}$, c. $31 \mathrm{delG}, \quad$ c. $472 \mathrm{G}>\mathrm{T}$, c. $564 \mathrm{dupG}, \quad$ c. $998 \mathrm{C}>\mathrm{T}$, c.1160A $>$ G, c.1199_1214dup16, c.1555delG, c.1891G $>$ A, c.1901T $>$ C and c.2027_2028delCT have been reported to be associated with $\mathrm{PCD}^{9,16-24}$ (Figure 5). Two novel $D N A A F 2$ variants were identified and confirmed to caused PCD in our patients through exome sequencing, immunofluorescence, and high-speed microscopy analysis. Situs inversus and male infertility have been previously reported due to DNAAF2 variants. No immunoreactivity for the ODA marker DNAI1 and DNAI2 in sperm flagella was observed for $D N A A F 2$-mutant individuals. Sperm flagellar length is significantly reduced in DNAAF2-mutant males. ${ }^{25}$ However, female infertility and scoliosis has not been investigated in patients with $D N A A F 2$ variant due to fallopian tubes and ependymal cells involved. Both Our patients both exhibited chronic sinusitis, bronchiectasis, reduced $\mathrm{nNO}$ production rate and female infertility, and one patient also presented with situs inversus and scoliosis.

Female infertility is reported less common than male infertility for PCD patients, which may relate to the

\section{Homozygous Regions for Proband1 Total $=146.97 \mathrm{Mb}$ (autosomes)}

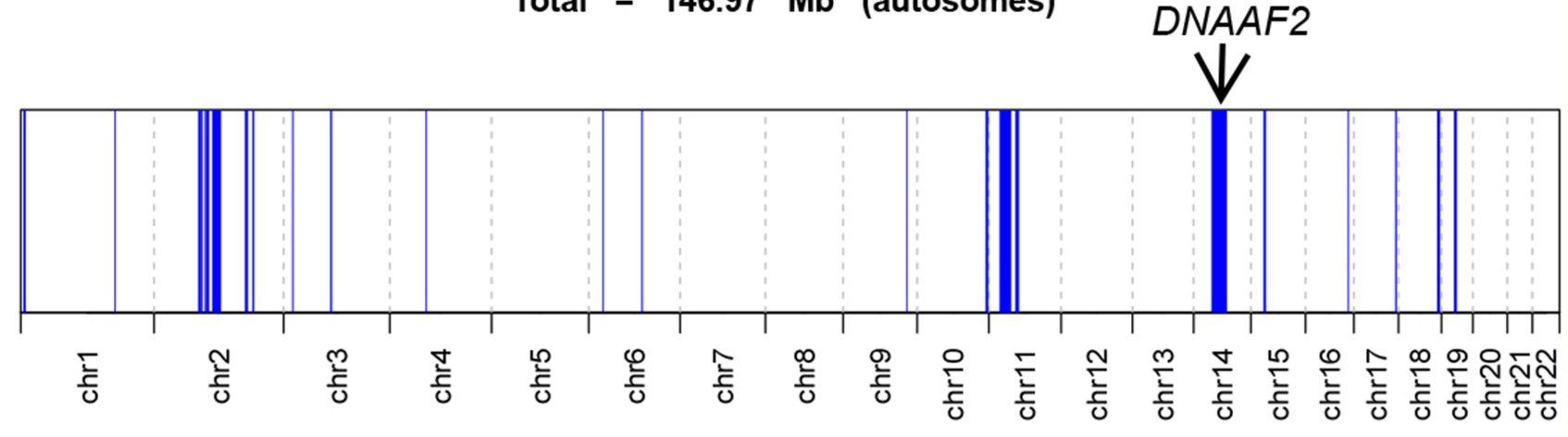

\section{Homozygous Regions for Proband2 Total $=343.42 \mathrm{Mb}$ (autosomes)}

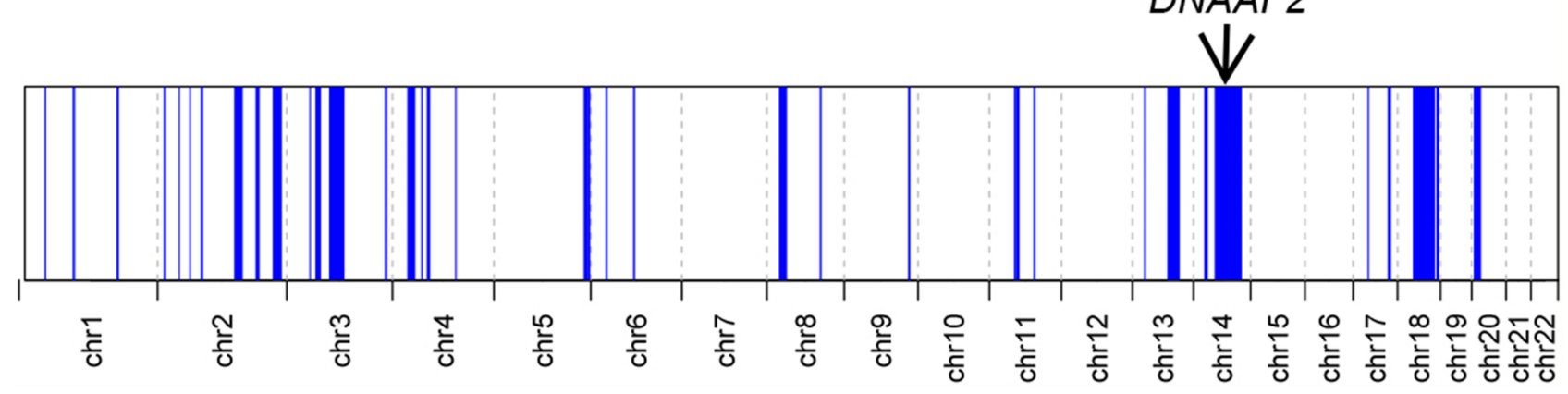

Figure 3 Homozygosity mapping using AutoMap software to detect regions of homozygosity (ROHs) larger than 2 Mb. ROHs are shown in blue for proband I and proband 2. The homozygous regions were shown in blue for proband I and proband 2 and the regions containing the DNAAF2 gene were marked with arrows. 


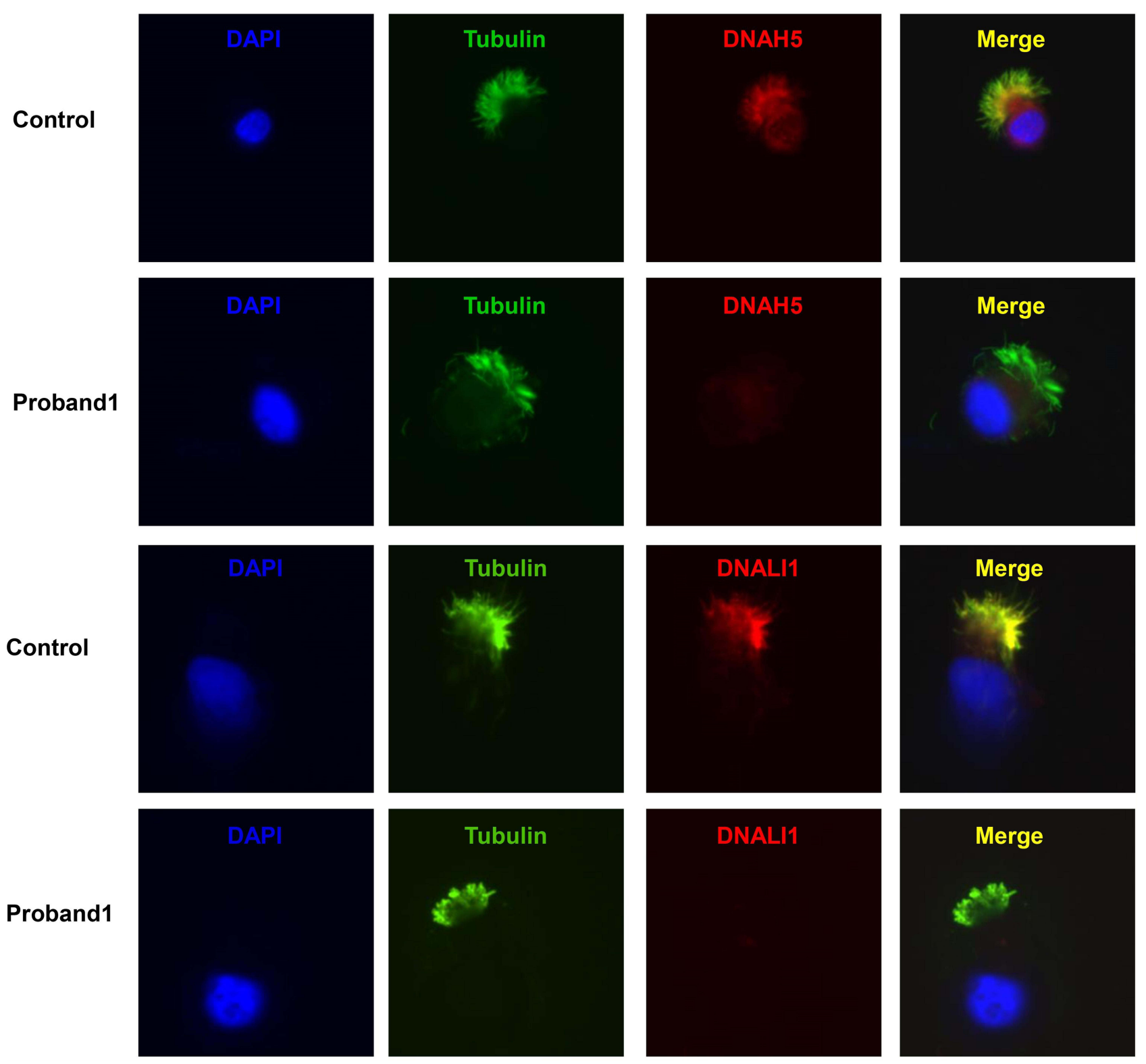

Figure 4 Immunofluorescence of nasal ciliated cells revealed the absence of DNAH5 and DNALII of proband I. Anti-acetylated tubulin monoclonal antibody was used to mark the ciliary axoneme. DNAH5 was used to label the outer dynein arm (ODA), DNALII was used to label the inner dynein arm (IDA) and DAPI was used to label the nuclei.

difficult in functional assessment of the cilia in fallopian heterogeneous in PCD, only a subset of PCD associated tubes. In one study, ${ }^{26} 22$ of 36 adult female patients with genes have been evaluated in the study. The phenotype of PCD presented with infertile. However, as the genetic fertility in patient with DNAAF2 variant was not

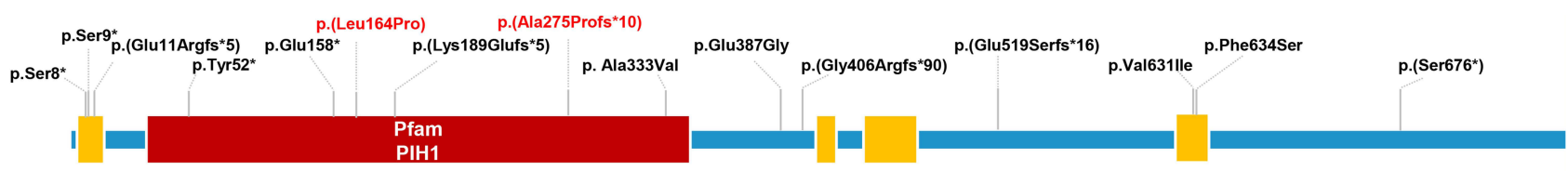

\section{Low complexity region}

Figure 5 DNAAF2 protein structure and the reported disease-causing variants of DNAAF2. The novel variants reported in this study are highlighted in red. 
established. The effect of cilia-related genes on female fertility may relate to the selective expression of genes in fallopian tubes. Cilia are located at the top of fallopian tube mucosa and is one of the main components of the mucosa. Coordinated ciliary movements in conjunction with muscle contractions transport egg cells through the fallopian tubes to the uterus, which is important to natural pregnancy. ${ }^{4,27}$ Human nasal cilia and fallopian tube cilia have similar structures. ${ }^{28}$ As female patients with other dynein assembly factor variants such as DNAAF1 and LRRC6 were $100 \%$ infertile in the cohort, ${ }^{26}$ we can infer that female infertility observed in proband 1 and proband 2 was due to $D N A A F 2$ deficiency and dysmotility of fallopian tube cilia. Further research was warrant to recapture and investigate the underlying mechanism of the phenotype in animal and human genetic studies.

Scoliosis was observed in serval animal models with PCD-related gene mutations, such as DNAAF4, ODAD3, $C C D C 40$, and $Z M Y N D 10^{6,29}$ However, scoliosis has rarely reported in patients with PCD. In 198 PCD patients collected by Schlösser et al, it was found that patients with Cobb angle greater than $10^{\circ}$ accounted for $8 \%$, but they only evaluated the chest radiographs and the genetic diagnosis was not analyzed. ${ }^{30}$ The etiology of idiopathic scoliosis remains unknown and the curvature of the spine is highly variable. ${ }^{7,31,32}$ The effect of abnormal cilia function is one of the possible reasons. Bearce et al believe that motile cilia, cerebrospinal fluid contacting neuron, the Reissner fiber and Urotensin neuropeptide signals constitute a "sensory organ" to sense the growth and development of the body axis. ${ }^{33}$ There is another hypothesis that the spine will follow its pre-existent rotational pattern if it decompensates into an idiopathic scoliosis. It means that scoliosis in adult PCD patients with situs inversus may also result from decompensation of the spine that due to situs inversus rather than as a single result of early ciliary movement. ${ }^{30}$ Although there are no reports mentioned scoliosis and cilia abnormality at the same time, the same gene is reported to be related to scoliosis or cilia abnormality in some articles. ${ }^{34-40}$ In our patient, DNAAF2 variant impaired cilia motility, leading to situs versus, and may be related to her scoliosis phenotype. In future, to explore the causes of scoliosis in patients with PCD will help to raise the public's attention to the spinal health, and carry out early intervention on spinal abnormality.

It should be noted that our study has examined only two patients with female infertility and one patient with scoliosis, and no intervention has been conducted. In vitro fertilization may be effective to treat infertility in female patients with PCD as previously reported. Although the phenotype of scoliosis has been observed in several PCD animal models, scoliosis has rarely been evaluated in human genetic studies. Whether DNAAF2 variant leads to scoliosis needs further validation.

In summary, we identified and validated the pathogenicity of two novel homozygous variants of DNAAF2, p. (Leu164Pro) and p.(Ala275Profs*10). Our findings expand the spectrum of DNAAF2 variants. Furthermore, we propose a link between female infertility, likely scoliosis, and DNAAF2 deficiency.

\section{Data Sharing Statement}

The data that support the findings of this study are available from the corresponding authors, Hong Luo and Ting Guo, upon reasonable request.

\section{Ethics Statement}

The studies involving human participants were reviewed and approved by the Review Board of the Second Xiangya Hospital of Central South University in China. Written informed consent to participate in this study was provided by the participants' legal guardian/next of kin. Written informed consent was obtained from the individual(s), and minor(s)' legal guardian/next of kin, for the publication of any potentially identifiable images or data included in this article.

\section{Acknowledgments}

We thank the patients and their families who participated in this study.

\section{Author Contributions}

All authors made a significant contribution to the work reported, whether that is in the conception, study design, execution, acquisition of data, analysis and interpretation, or in all these areas; took part in drafting, revising or critically reviewing the article; gave final approval of the version to be published; have agreed on the journal to which the article has been submitted; and agree to be accountable for all aspects of the work.

\section{Funding}

This study was supported by the National Natural Science Foundation of China (81470202, 81770002 and 82070003 to H.L, 81900002 to T.G), Natural Science Foundation of Hunan Province, China (2020JJ5805 to T.G, 2021JJ30943 
to H.L), the Hunan Province Health Commission Scientific Research Project (B2019159 to H.L), the Science and Technology Program of Changsha, China (kq1901120 to H.L), Xiangya Clinical Big Data System Construction Project in Pulmonary Inflammatory Disease of Central South University, and the National Key Clinical Specialty Construction Projects of China.

\section{Disclosure}

The authors report no conflicts of interest in this work.

\section{References}

1. Mirra V, Werner C, Santamaria F. Primary ciliary dyskinesia: an update on clinical aspects, genetics, diagnosis, and future treatment strategies. Front Pediatr. 2017;5:135. doi:10.3389/fped.2017.00135

2. Knowles MR, Zariwala M, Leigh M. Primary ciliary dyskinesia. Clin Chest Med. 2016;37(3):449-461. doi:10.1016/j.ccm.2016.04.008

3. Flume PA, Chalmers JD, Olivier KN. Advances in bronchiectasis: endotyping, genetics, microbiome, and disease heterogeneity. Lancet. 2018;392(10150):880-890. doi:10.1016/S0140-6736(18)31767-7

4. Wallmeier J, Nielsen KG, Kuehni CE, et al. Motile ciliopathies. Nat Rev Dis Primers. 2020;6(1):77.

5. Wang R, Yang D, Guo T, et al. Case report: identification of a novel ODAD3 variant in a patient with primary ciliary dyskinesia. Front Genet. 2021;12:652381. doi:10.3389/fgene.2021.652381

6. Grimes DT, Boswell CW, Morante NFC, et al. Zebrafish models of idiopathic scoliosis link cerebrospinal fluid flow defects to spine curvature. Science. 2016;352(6291):1341-1344. doi:10.1126/ science.aaf6419

7. Aebi M. The adult scoliosis. Eur Spine J. 2005;14(10):925-948. doi:10.1007/s00586-005-1053-9

8. Horani A, Ustione A, Huang T, et al. Establishment of the early cilia preassembly protein complex during motile ciliogenesis. Proc Natl Acad Sci U S A. 2018;115(6):E1221-E1228. doi:10.1073/ pnas. 1715915115

9. Omran H, Kobayashi D, Olbrich $H$, et al. Ktu/PF13 is required for cytoplasmic pre-assembly of axonemal dyneins. Nature. 2008;456 (7222):611-616. doi:10.1038/nature07471

10. Shapiro AJ, Dell SD, Gaston B, et al. Nasal nitric oxide measurement in primary ciliary dyskinesia. A technical paper on standardized testing protocols. Ann Am Thorac Soc. 2020;17(2):e1-e12. doi:10.1513/AnnalsATS.201904-347OT

11. Wang K, Li M, Hakonarson H. ANNOVAR: functional annotation of genetic variants from high-throughput sequencing data. Nucleic Acids Res. 2010;38(16):e164. doi:10.1093/nar/gkq603

12. Richards S, Aziz N, Bale S, et al. Standards and guidelines for the interpretation of sequence variants: a joint consensus recommendation of the American college of medical genetics and genomics and the association for molecular pathology. Genet Med. 2015;17 (5):405-423. doi:10.1038/gim.2015.30

13. Quinodoz M, Peter VG, Bedoni N, et al. AutoMap is a high performance homozygosity mapping tool using next-generation sequencing data. Nat Commun. 2021;12(1):518. doi:10.1038/s41467-02020584-4

14. Lucas JS, Davis SD, Omran H, et al. Primary ciliary dyskinesia in the genomics age. Lancet Respir Med. 2020;8(2):202-216. doi:10.1016/ S2213-2600(19)30374-1

15. Goutaki M, Meier AB, Halbeisen FS, et al. Clinical manifestations in primary ciliary dyskinesia: systematic review and meta-analysis. Eur Respir J. 2016;48(4):1081-1095. doi:10.1183/13993003.00736-2016
16. Sun M, Zhang Y, Wang Y, et al. Novel compound heterozygous DNAAF2 mutations cause primary ciliary dyskinesia in a Han Chinese family. J Assist Reprod Genet. 2020;37(9):2159-2170. doi:10.1007/s10815-020-01859-7

17. Zhu N, Welch CL, Wang J, et al. Rare variants in SOX17 are associated with pulmonary arterial hypertension with congenital heart disease. Genome Med. 2018;10(1):56. doi:10.1186/s13073018-0566-x

18. Paff T, Kooi IE, Moutaouakil Y, et al. Diagnostic yield of a targeted gene panel in primary ciliary dyskinesia patients. Hum Mutat. 2018;39(5):653-665. doi:10.1002/humu.23403

19. Emiralioğlu N, Taşkıran EZ, Koşukcu C, et al. Genotype and phenotype evaluation of patients with primary ciliary dyskinesia: first results from Turkey. Pediatr Pulmonol. 2020;55(2):383-393. doi:10.1002/ppul.24583

20. Alhathal N, Maddirevula S, Coskun S, et al. A genomics approach to male infertility. Genet Med. 2020;22(12):1967-1975. doi:10.1038/ s41436-020-0916-0

21. Kim RH, A. Hall D, Cutz E, et al. The role of molecular genetic analysis in the diagnosis of primary ciliary dyskinesia. Ann Am Thorac Soc. 2014;11(3):351-359. doi:10.1513/AnnalsATS.201306194OC

22. Capalbo A, Valero RA, Jimenez-Almazan J, et al. Optimizing clinical exome design and parallel gene-testing for recessive genetic conditions in preconception carrier screening: translational research genomic data from 14,125 exomes. PLoS Genet. 2019;15(10):e1008409. doi:10.1371/journal.pgen.1008409

23. LaDuca H, Farwell KD, Vuong H, et al. Exome sequencing covers $>98 \%$ of mutations identified on targeted next generation sequencing panels. PLoS One. 2017;12(2):e0170843. doi:10.1371/journal. pone. 0170843

24. Blanchon S, Legendre M, Bottier M, et al. Deep phenotyping, including quantitative ciliary beating parameters, and extensive genotyping in primary ciliary dyskinesia. J Med Genet. 2020;57(4):237-244. doi:10.1136/jmedgenet-2019-106424

25. Aprea I, Raidt J, Höben IM, et al. Defects in the cytoplasmic assembly of axonemal dynein arms cause morphological abnormalities and dysmotility in sperm cells leading to male infertility. PLoS Genet. 2021;17(2):e1009306. doi:10.1371/journal.pgen.1009306

26. Vanaken GJ, Bassinet L, Boon M, et al. Infertility in an adult cohort with primary ciliary dyskinesia: phenotype-gene association. Eur Respir J. 2017;50(5):1700314. doi:10.1183/13993003.00314-2017

27. Lyons RA, Saridogan E, Djahanbakhch O. The reproductive significance of human Fallopian tube cilia. Hum Reprod Update. 2006;12 (4):363-372. doi:10.1093/humupd/dml012

28. Raidt J, Werner C, Menchen T, et al. Ciliary function and motor protein composition of human fallopian tubes. Hum Reprod. 2015;30 (12):2871-2880. doi:10.1093/humrep/dev227

29. Kobayashi D, Asano-Hoshino A, Nakakura T, et al. Loss of zinc finger MYND-type containing 10 (zmynd10) affects cilia integrity and axonemal localization of dynein arms, resulting in ciliary dysmotility, polycystic kidney and scoliosis in medaka (Oryzias latipes). Dev Biol. 2017;430(1):69-79. doi:10.1016/j.ydbio.2017.08.016

30. Schlösser TPC, Semple T, Carr SB, et al. Scoliosis convexity and organ anatomy are related. Eur Spine J. 2017;26(6):1595-1599. doi:10.1007/s00586-017-4970-5

31. Cheng JC, Castelein RM, Chu WC, et al. Adolescent idiopathic scoliosis. Nat Rev Dis Primers. 2015;1(1):15030. doi:10.1038/ nrdp. 2015.30

32. Oliazadeh N, Gorman KF, Eveleigh R, et al. Identification of elongated primary cilia with impaired mechanotransduction in idiopathic scoliosis patients. Sci Rep. 2017;7(1):44260. doi:10.1038/srep44260

33. Bearce EA, Grimes DT. On being the right shape: roles for motile cilia and cerebrospinal fluid flow in body and spine morphology. Semin Cell Dev Biol. 2021;110:104-112. doi:10.1016/j. semcdb.2020.07.005 
34. Zhou S, Xie Y, Tang J, et al. FGFR3 deficiency causes multiple chondroma-like lesions by upregulating hedgehog signaling. PLoS Genet. 2015;11(6):e1005214. doi:10.1371/journal.pgen.1005214

35. Nita A, Abraham SP, Krejci P, et al. Oncogenic FGFR fusions produce centrosome and cilia defects by ectopic signaling. Cells. 2021;10(6):1445. doi:10.3390/cells10061445

36. Makrythanasis P, Temtamy S, Aglan MS, et al. A novel homozygous mutation in FGFR3 causes tall stature, severe lateral tibial deviation, scoliosis, hearing impairment, camptodactyly, and arachnodactyly. Hum Mutat. 2014;35(8):959-963. doi:10.1002/humu.22597

37. Gao C, Chen BP, Sullivan MB, et al. Micro CT analysis of spine architecture in a mouse model of scoliosis. Front Endocrinol. 2015;6:38. doi:10.3389/fendo.2015.00038
38. Baschal EE, Swindle K, Justice CM, et al. Sequencing of the TBX6 gene in families with familial idiopathic scoliosis. Spine Deform. 2015;3(4):288-296. doi:10.1016/j.jspd.2015.01.005

39. Fei $\mathrm{Q}, \mathrm{Wu} \mathrm{Z}$, Wang $\mathrm{H}$, et al. The association analysis of TBX6 polymorphism with susceptibility to congenital scoliosis in a Chinese Han population. Spine. 2010;35(9):983-988. doi:10.1097/ BRS.0b013e3181bc963c

40. Hadjantonakis A-K, Pisano E, Papaioannou VE, Zwaka T. Tbx6 regulates left/right patterning in mouse embryos through effects on nodal cilia and perinodal signaling. PLoS One. 2008;3(6):e2511. doi:10.1371/journal.pone.0002511

\section{Publish your work in this journal}

Pharmacogenomics and Personalized Medicine is an international, peer-reviewed, open access journal characterizing the influence of genotype on pharmacology leading to the development of personalized treatment programs and individualized drug selection for improved safety, efficacy and sustainability. This journal is indexed on the American Chemical Society's Chemical Abstracts Service (CAS). The manuscript management system is completely online and includes a very quick and fair peer-review system, which is all easy to use. Visit http://www.dovepress.com/testimonials.php to read real quotes from published authors. 